Nanotechnology

\section{Nanotubes get}

\section{sorted}

Science doi:10.1126/science.1086534 (2003) ${ }^{1}$

J. Phys. Chem. doi:10.1021/jp027664a (2003) ${ }^{2}$

Researchers wanting to use carbon nanotubes as components for nanoscale electronics face the problem that standard synthetic methods produce a combination of metallic and semiconducting tubes. The differing electronic properties are a consequence of only very subtle differences in molecular structure, making the two types hard to separate. But now there is a choice of two techniques for doing this.

Ralph Krupke et al. ${ }^{1}$ make use of the different dielectric constants of metallic and semiconducting nanotubes. In an aqueous suspension, the two types respond to an alternating electric field by moving in opposite directions along the electric-field gradient. The researchers achieved $80 \%$ enrichment in metallic nanotubes, which are normally present in a nanotube sample in a $1: 2$ ratio.

Michael S. Strano et al. ${ }^{2}$ propose a chemical separation based on $\mathrm{pH}$ titration. They report that the readiness with which the carbon walls of nanotubes are protonated depends on the electronic band gap. Metallic tubes (which have a band gap of zero) can be protonated at near-neutral $\mathrm{pH}$, but increasingly acidic solutions are needed as the band gap increases (as it does in semiconducting nanotubes). The protonation is reversible at alkaline pH. This should allow continuous 'fractionation' of nanotubes according to their band gap.

Philip Ball

\section{Cancer}

\section{From breast to bone}

Cancer Cell 3, 537-549 (2003)

It is not clear how metastasis - the spread of tumour cells to distant organs - occurs. Some believe that most cells in a primary tumour carry a set of metastasis-causing genetic alterations. But from their study of human breast cancer cells, Yibin Kang et al. provide evidence to the contrary.

Most patients with advanced breast cancer develop secondary tumours in their bones. Kang et al. found that, after inoculating mice with cells from a human breast cancer line, $30 \%$ of the animals also went on to develop bone tumours. From these tumours, the authors pulled out a subpopulation of highly metastatic cells and studied their genetic profile. The cells overexpressed several genes, including those involved in forming new blood vessels and the ability to home in on and invade bone. This suggests that key genes may influence the environment around a tumour in favour of metastasis.
Notably, however, only a fraction of the original breast cancer cells had the genetic predisposition to infiltrate bone.

This work reveals a firm link between bone metastasis and the expression of several genes that have previously only been correlated with this process. Helen R. Pilcher

\section{Earth science}

\section{High expectations}

Geophys. Res. Lett. doi:10.1029/2003GL017708 (2003)

Arrays of sensors such as the Southern

California Integrated GPS Network

(SCIGN), designed for monitoring

earthquake-prone faults in southern

California, show promise for studying the ionosphere, according to E. Calais and colleagues.

GPS - Global Positioning System sensors rely on radio signals sent by satellites: as the signals pass through the ionosphere, electromagnetic disturbances in this region can influence signal timing. Events on the ground or in the electrically neutral atmosphere produce upwardmoving gravity waves, which affect electron density in the ionosphere.

Calais et al. now show that, given a dense enough array of sensors (there are 250 in the SCIGN), it is possible to overcome the small signal-to-noise ratio of such events and identify the direction and speed of the resulting ionospheric disturbances. They were looking for, but failed to find, a 'fingerprint' left in the data by a particular earthquake. Serendipitously, however, they identified other perturbations which they suggest may possibly have been caused by powerful turbulent activity in the lower atmosphere. It is early days. But the approach could prove to be a valuable one in investigating fine-scale atmospheric processes with previously unachievable spatial and temporal resolution. Tom Clarke

\section{Quantum physics \\ How cold can you go?}

Phys. Rev. A 67, 061401(R) (2003)

Bose-Einstein condensation (BEC), creating an exotic and potentially useful quantum state of matter, can be achieved with an unorthodox cooling method, say D. Ivanov and colleagues.

BEC typically requires temperatures of a few billionths of a kelvin. Such cooling is attained in a gas of trapped atoms by, for example, bouncing laser light off the atoms to slow them down. But this and other cooling methods have limitations (for example, in the types of atoms that can be cooled) that could be avoided by stochastic cooling. Here a laser beam 'finds' energetic atoms in a gas cloud and adjusts their momenta selectively — rather like Maxwell's demon. In effect, the beam measures the momentum (or energy) distribution in a region of gas and then exerts a feedback to shift the mean momentum to zero.

Implementations of stochastic cooling have been proposed previously, but it wasn't clear how cold it could get. Ivanov et al. have calculated the quantum limits on the procedure, and find that it can reach the BEC temperature of an atomic gas, despite the heating caused by quantum fluctuations near the condensation point.

Philip Bal

\section{Developmental biology}

\section{Fish in pinstripe suits}

Development 130, 3447-3457 (2003)

Adult zebrafish have stripes running from head to tail. These are formed by alternating rows of two types of pigment cell — blue melanophores and yellow xanthophores. Florian Maderspacher and Christiane Nüsslein-Volhard now show that the interactions between these cells determine the razor-sharp look of the stripes.

The authors first found that pigment cells do not simply 'colour in' a predefined pattern. Stripes form only if both cell types are present and in close proximity. To look for factors that control the cellular interactions at play, the authors then scrutinized two zebrafish mutants, obelix and leopard (see picture).

Obelix fish have fewer and wider stripes than normal, and, in severe cases, blurred stripe boundaries. The gene affected in obelix mutants appears to act only in melanophores, promoting their aggregation. This is necessary to keep the two cell types spatially separated, so affecting the integrity of boundaries. The leopard gene is required by both cell types, and its absence in either transforms stripes into spots. The gene seems to control the interactions between cells of the same type and between those of different types, thereby regulating the shape of the boundary.

The molecules encoded by the obelix and leopard genes are not known, however, nor are they the only factors involved in stripe formation.

Marie-Thérèse Heemels

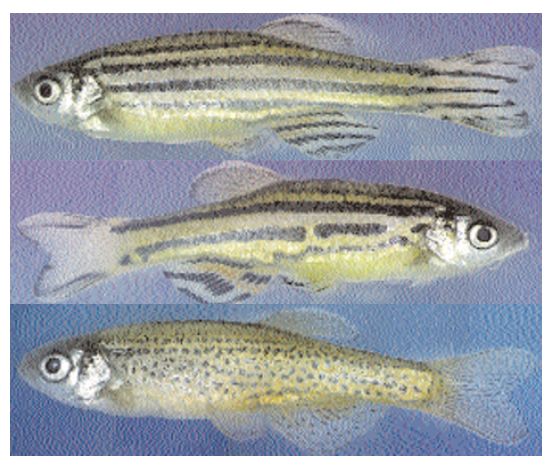

How the zebrafish... From top, a normally striped fish, and obelix and leopard mutants. 